\title{
Temperature dependence of magnetic resonance sensors for embedding into constructed wetlands
}

\section{Theodore Hughes-Riley ${ }^{1}$, Michael I. Newton ${ }^{1}$ and Robert H. Morris ${ }^{1, *}$}

1 School of Science and Technology. Nottingham Trent University, United Kingdom: theodore.hughesriley@ntu.ac.uk; michael.newton@ntu.ac.uk

* Author to whom correspondence should be addressed; E-Mail: rob.morris@ntu.ac.uk; Tel.: +(44) 01158483315

Received: / Accepted: / Published:

\begin{abstract}
Constructed wetlands are an environmentally considerate means of water purification. One critical parameter to monitor in a wetland system is clogging of pores within the gravel matrix, as this limits the viable lifetime of the system. It has previously been observed that magnetic resonance (MR) relaxation measurements, $\mathrm{T}_{1}$ and $\mathrm{T}_{2}{ }^{\text {eff }}$, can be used to characterise the clogging state. Various open-geometry MR sensors have been constructed using permanent neodymium magnets with the view of long-term embedding as part of the EU FP7 project ARBI (Automated Reed Bed Installations). The ultimate aim is to monitor clogging levels over the lifetime of the reed bed using MR techniques. One issue with taking various MR measurements over a time scale on the order of years is that temperature fluctuations will alter the magnetic field strength produced by the sensors constituent magnets. While the RF transmit-receive circuit has been built so that MR can still be conducted at a range of frequencies, this will result in poor RF excitation if the magnetic field strength shifts significantly. This work investigates the effect that temperature has on the an MR sensor intended for embedding, to determine whether received signal intensity is compromised significantly at large temperature changes.
\end{abstract}

Keywords: constructed wetlands; Nuclear Magnetic Resonance; clogging state; spin lattice relaxation; spin-spin relaxation; embedded sensor; temperature dependence

\section{Introduction}


Constructed wetlands have proliferated across the world in recent years as an environmentally conscious waste-water treatment system. A prohibitive factor to wetland units is their limited operational lifetimes with as little as 10 years seeing clogging in the pores of the wetland gravel matrix becoming significant enough to no longer allow for water treatment. Reconditioning of a wetland is a time consuming and expensive process, and should therefore be avoided if possible. As a result it is important to monitor clogging level of beds to allow for optimal wetland management.

No real time techniques currently exist to monitor clogging. One method to determine clog state is to investigate the hydrodynamic pathways, as the motion of liquid will be retarded in areas where the pores in the gravel matrix are blocked. This can be performed using a tracer dye (such as Rhodamine WT) and monitoring at different positions in the reed bed using a fluorimeter, [1; 2].

Magnetic resonance has been shown to be effected in determining the clog state through two parameters $\mathrm{T}_{1}$ and $\mathrm{T}_{2}{ }^{\text {eff }}$ in a laboratory setting [3-5]. It has been proposed that low-field MR sensors built using permanent $\mathrm{NdFeB}$ magnets can be embedded for long term monitoring of clog state and tested in a controlled environment[3]. While useful for proving the viability of the technique, there are additional considerations when monitoring an operational wetland over long time scales.

One important factor is temperature. Extremes of temperature can cause irreversible losses in magnetization [6]. Smaller increases in the temperature of a substance causes the electrons to move further away from their nucleus, increasing its orbital diameter. Therefore electrons will have a decreased influence on their counterparts (as they will come into contact less frequently) thus decreasing the magnetic flux.

Literature dealing with reversible flux losses in $\mathrm{NbFeB}$ magnets show small changes at low temperatures [6] compared to the overall magnetic field strength, however these changes may still be significant for MR where a change as low as $1 \mathrm{mT}$ may be enough to lose all signal.

This work investigates two sensor designs; a Helmholtz arrangement[3] and a unilateral sensor using the stray field of a Halbach arrangement [4; 5]. The RF transmit-receive coils have been built in such a way that they can excite a range of frequencies without the need for the tuning or matching values to be altered, however a significant change in the magnetic field might seriously alter the acquired MR signal intensity. Ultimately the strength of MR signal at different temperatures will be examined to determine the viability of these sensors under the conditions they will encounter in situ.

\section{Experimental Section}

\subsection{MR sensor designs}

Two MR sensors were used in this study. The first was a Helmholtz configuration similar to a design previously presented by Morris et al.[3]. Two N42 neodymium magnets (height $=20 \mathrm{~mm}$, radius $=17$ $\mathrm{mm}$; Magnet Monster, Germany) were arranged with parallel magnetisation and spaced by $20 \mathrm{~mm}$ generating a uniform magnetic field in the magnet spacing (325 mT at $292 \mathrm{~K}$ ), as shown in Figure 1a. Steel disks were used on the faces of the magnets to improve field uniformity.

The RF transmit-receive coil took the shape of an eight-turn solenoid attached to parallel-series tuning boards with two variable capacitors (12-100 pF; Johanson Manufacturing, NJ, USA) and a 55 pF fixed tuning capacitor, completing a resonant circuit operating at $13.87 \mathrm{MHz}$. 
The second MR sensor used the stray magnetic field from a four magnet Halbach array, inspired by work by Chang et al.[7], to produce a region of uniform magnetic field slightly above the magnet faces (Figure 1b). The sensor used four N42 neodymium magnets with the dimensions $30 \mathrm{x} 40 \mathrm{x} 40 \mathrm{~mm}^{3}$ with their magnetic polarization along the $30 \mathrm{~mm}$ axis (First4Magnets, UK). Copper tape was placed over the surfaces of the magnets to limit RF loading. $7.5 \mathrm{~mm}$ steel plates were attached to the bottom of the magnets to help reduce the field gradient to $15.2 \mathrm{~T} / \mathrm{m}$ normal to the surface of the magnets.

The RF transmit-receive coil was made from two turns of enameled copper wire (Rowan Cable, UK)[8]. This was attached to a parallel-series tuning board similar to the one described above with an additional $1680 \mathrm{pF}$ for tuning and $390 \mathrm{pF}$ for matching to achieved the required resonance at $10.3 \mathrm{MHz}$.

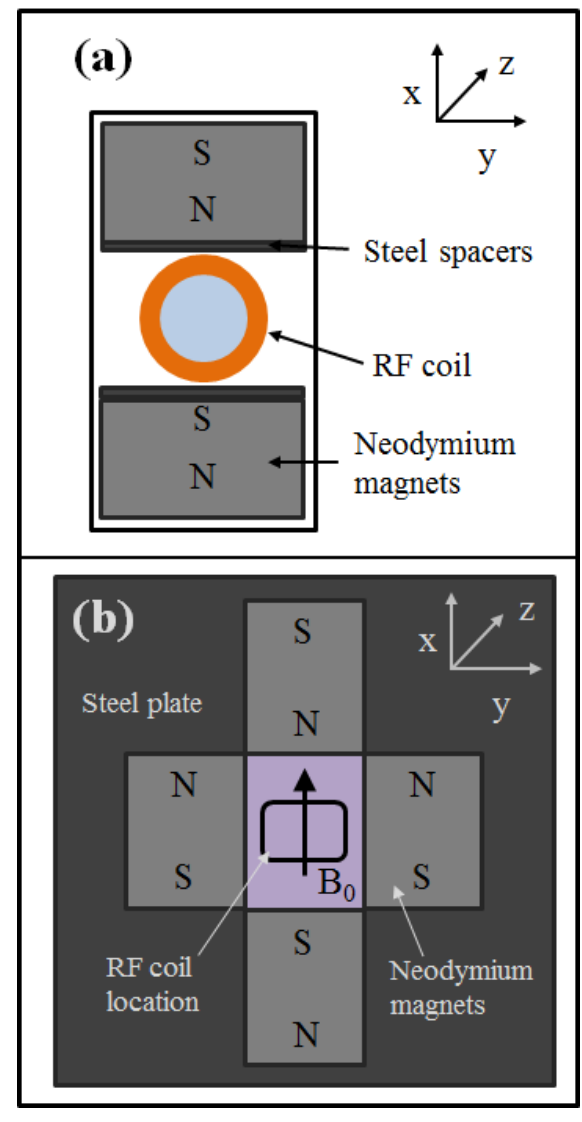

Figure 1. The two sensor designs used in this work. Figure is not to scale. (a) Helmholtz sensor. Two magnets were used to generate a uniform region of field in the magnet gap. Steel disks were used on each to reduce the magnetic field gradient. (b) Halbach sensor. Four magnets were used to generate a uniform region of field just above the magnet gap. Magnets were cover in copper tape to limit RF loading. Steel plates were place on the base of the magnets to reduce the field gradient.

\subsection{MR protocol}

MR was performed using a commercial Kea 2 spectrometer (Magritek, New Zealand) running Prospa 3.12 software. The only MR sequence used in this work was a Carr Purcell Meiboom Gill (CPMG) [9] sequence. For signal intensity measurements, multiple echoes were summed to reduce the number of averages required. MR experiments were run on a 'thick' wetland sludge (supplied by ARM limited, Rugeley, UK) representative of a reed bed close to the end of its operational lifetime.

\subsection{Inducing temperature changes}

To heat the magnet assemblies, the sensors were left in a convection oven (Binder, Germany) set to $60{ }^{\circ} \mathrm{C}$ for around two hours, heating the magnets to approximately $40{ }^{\circ} \mathrm{C}$. Temperature was measured 
using a type-K thermocouple attached to an electronic thermometer to read off values (Comark, UK). The sensor was left to cool naturally by equilibrating with room temperature (typically $20^{\circ} \mathrm{C}$ ).

To cool the sensors, a freezer (Norfrost, UK) set to below $-16{ }^{\circ} \mathrm{C}$ was used. The sensors were left overnight (approximately 14 hours), with the Halbach sensor cooling to $-16^{\circ} \mathrm{C}$. The sensors were then left to equilibrate with room temperature.

Errors in the temperature are taken as the variation in the temperature from the start of the experiment to the end, in addition to the $0.5^{\circ} \mathrm{C}$ accuracy of the electronic thermometer for both readings.

\section{Results and Discussion}

Signal intensity as a function of temperature was investigated between $-16^{\circ} \mathrm{C}$ and $40^{\circ} \mathrm{C}$ for the Halbach sensor (Figure 2), and between $43^{\circ} \mathrm{C}$ and $-10^{\circ} \mathrm{C}$ for the Helmholtz sensor (Figure 3). Measurements were taken at a selection of different temperatures to get a fair representation over the range of interest.

For the Halbach sensor it can be observed that there is a weak linear relationship between acquired signal intensity and temperature. A linear fitting was applied showing a gradient of $-2.8 \times 10^{-5} \pm 5 \times 10^{-6}$ $\mu \mathrm{V} /{ }^{\circ} \mathrm{C}$.

Deviation from this is likely due to errors in the signal intensity introduced by insufficient averages. The number of averages was limited to 2048, and repetition time limited to only $1 \mathrm{x} \mathrm{T}_{1}$ to keep the experiments short ( $<15$ minutes), as over longer time scales the temperature changed too significantly.

The relationship itself is likely a result of the flux density of the magnets increasing at lower temperatures; as the electrons make tighter orbits of their nuclei and therefore exert a greater influence on one another. An increase in flux density will lower the position of the excited region of the sensor, bringing it closer to the RF coil making detection of the spins easier.

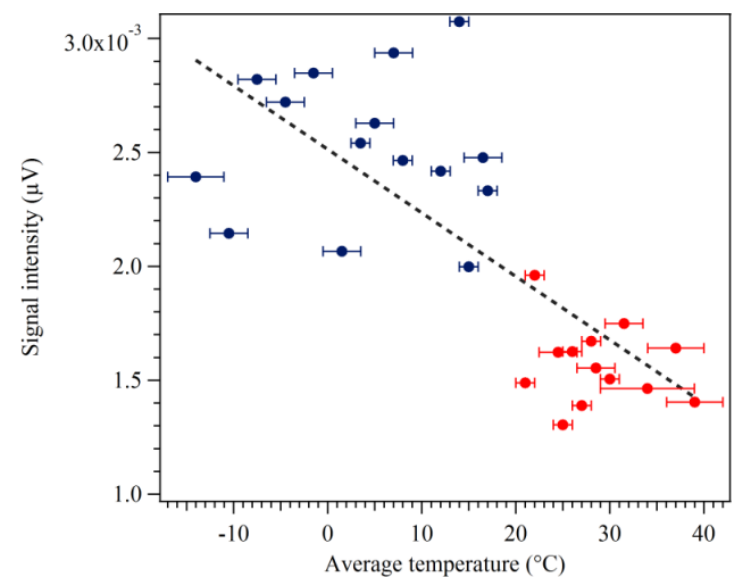

Figure 2. Signal intensity as a function of temperature for Halbach sensor on a thick wetland sample. Red indicates measurements where the magnet was heated and left to cool, while blue shows measurements were the magnet was cooled and allowed to heat. CPMG trains 64 echoes were taken and summed to reduce averages $\left(\tau_{\mathrm{E}}=100 \mu \mathrm{s}, 400 \mathrm{~ms}\right.$ repetition time, 2048 averages).

Figure 3 clearly shows that there is an optimal operating temperature for the Helmholtz sensor (22 \pm $2{ }^{\circ} \mathrm{C}$ ), which is very close to the temperature that the sensor was designed and constructed at. Drop-off in signal intensity at both high and low temperatures is likely due to the sensitive region of the sensor moving as the magnetic field changes. The sensor will be at its optimal operating conditions when the magnetic field at the center of the solenoid is the same as the RF sensors resonant frequency. 
Temperature changes effect the magnetic field and this will move the sensitive region of the sensor, making less of the volume of the solenoid sensitive.

Despite the major drop in signal intensity MR measurements are still possible at both low and high temperatures and as a result successful operation of a sensor outside over a long period should be possible.

Figure 3. Signal intensity as a function of temperature for Helmholtz sensor on a thick wetland sample. Red indicates measurements where the magnet was heated and left to cool, while blue shows measurements where the magnet was cooled and then warmed. CPMG trains with 128 echoes were taken and summed to reduce averages $\left(\tau_{\mathrm{E}}=100 \mu \mathrm{s}, 400 \mathrm{~ms}\right.$ repetition time, 512 averages).

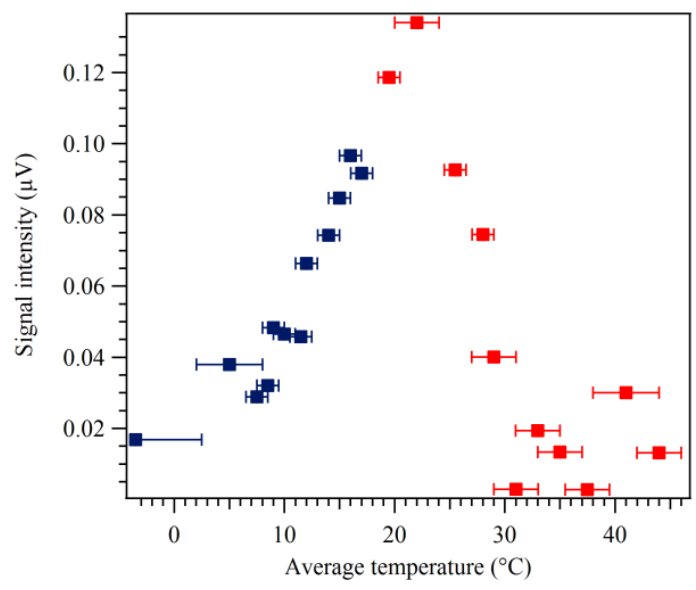

\section{Conclusions/Outlook}

This work validates the ability to operate MR sensors over a wide range of temperatures covering the maximum likely scope that a wetland deployed in Europe would experience. For the Halbach sensor, signal intensity has been seen to have a weak linear relationship with temperature (with a gradient of $2.8 \times 10^{-5} \pm 5 \times 10^{-6} \mu \mathrm{V} /{ }^{\circ} \mathrm{C}$ ), favoring cold temperatures. This may affect the number of signal averages required for wetlands deployed to warmer climates.

A Helmholtz MR sensor was also investigated. This sensor was seen to operate best at close to laboratory temperatures $\left(22 \pm 2{ }^{\circ} \mathrm{C}\right)$. However this sensor was still able to collect MR measurements at high and low temperatures, validating its use in a wetland system long-term.

Further work will also see a probe embedded long term, where measurements can be taken over a number of months as the wetland clogs. This should be possible in spite of seasonal temperature variations.

\section{Acknowledgments}

The research leading to these results was funded by the European Union's Seventh Framework Programme managed by the REA - Research Executive Agency http://ec.europa.eu/research/rea(FP7/2007_2013) under project reference 606326.

\section{Author Contributions}

This work involved three authors. M.I. Newton and R.H. Morris proposed the experiments, and reviewed and edited the manuscript. T. Hughes-Riley collected and processed the data and wrote the paper. 


\section{Conflicts of Interest}

The authors declare no conflict of interest.

\section{References and Notes}

1. Lin, A. Y. C.; Debroux, J. F.; Cunningham, J. A.; Reinhard, M. Comparison of rhodamine WT and bromide in the determination of hydraulic characteristics of constructed wetlands. Ecological Engineering 2003, 20(1), 75-88.

2. Knowles, P. R.; Griffin, P.; Davies, P. A. Complementary methods to investigate the development of clogging within a horizontal sub-surface flow tertiary treatment wetland. Water research 2010, 44(1), 320-330.

3. Morris, R. H.; Newton, M. I.; Knowles, P. R.; Bencsik, M.; Davies, P. A.; Griffin, P.; McHale, G. Analysis of clogging in constructed wetlands using magnetic resonance. Analyst 2011, 136(11), 2283-2286.

4. Hughes-Riley, T; Newton, M.I.; Webber, J.B.; Pigaguat, J.; Ugetti, E.; Garcia, J.; Morris, R.H. Automated Reed Bed Installations. The 12th International Bologna Conference on Magnetic Resonance in Porous Media (MRPM12), Wellington, New Zealand, 9th- 13th/02/2014.

5. Hughes-Riley, T; Webber, J.B.; Newton, M.I.; Morris, R.H. Magnetic resonance relaxation measurements using open-geometry sensors to assess the clog state of constructed wetlands. Diffusion Fundamentals, under review.

6. Clegg, A. G.; Coulson, I. M.; Hilton, G.; Wong, H.Y. The temperature stability of NdFeB and NdFeBCo magnets. Magnetics, IEEE Transactions on 1991, 26(5), 1942-1944.

7. Chang, W.H.; Chen, J.H.; Hwang, L.P. Single-sided mobile NMR with a Halbach magnet. Magnetic resonance imaging 2006, 24(8), 1095-1102.

8. Perlo, J. Magnets and Coil for Single-Sided NMR. In Single-sided NMR; Casanova, F., Perlo, J., Blümich, B., Eds.; Springer: Heidelberg, Germany, 2011; pp. 102-103.

9. Meiboom, S.; Gill, D. Modified spin-echo method for measuring nuclear relaxation times. Review of scientific instruments 1958, 29(8), 688-691.

(C) 2014 by the authors; licensee MDPI, Basel, Switzerland. This article is an open access article distributed under the terms and conditions of the Creative Commons Attribution license (http://creativecommons.org/licenses/by/3.0/). 